\section{Krebsnachsorge: Programm für gestärkte Herzen}

Krebspatienten haben sich häufig sportliche Aktivität während der belastenden Tumortherapie abgewöhnt - mit neuen Gefahren für ihre Gesundheit. Ob ein Trainingsprogramm mit psychologischer Intervention helfen könnte?

V iele Krebsüberlebende pflegen einen überwiegend sitzenden Lebensstil und haben eine verminderte kardiovaskuläre Reservekapazität, wie sie sich etwa im Wert der höchsten Sauerstoffaufnahme unter Belastung $\left(\mathrm{VO}_{2 \text { peak }}\right)$ zeigt. Ihr Lebensstil kann den Patienten somit wieder schwere Gesundheitsprobleme bereiten und die Mortalität erhöhen.

In einer aktuellen randomisierten Studie evaluierten dänische Kollegen daher den Effekt eines 12-monatigen Rehabilitationsprogramms, das sportliche Aktivität in den Mittelpunkt stellte. 214 Überlebende einer Tumorerkrankung wurden nicht nur nach ihrer körperlichen Aktivität gefragt; es wurde auch die kardiorespiratorische Fitness in Form von $\mathrm{VO}_{2 \text { peak }}$ während eines Tests auf dem Fahrradergometer gemessen. Die Hälfte erhielt zwölf Monate lang ein Rehabilitationsprogramm namens
Copenhagen Physical Activity after Cancer Treatment (PACT: einmal wöchentlich 90-minütiges körperliches Training in der Gruppe, insgesamt drei Einzelund sechs Gruppensitzungen mit einem geschulten Psychologen). Die übrigen Patienten bekamen lediglich drei 15-minütige Gesundheitssitzungen, in denen mit ihnen über ihre persönliche Fitness und den Wert körperlicher Aktivität gesprochen wurde. Ziel war es, die Probanden dazu anzuregen, mindestens drei Stunden pro Woche Sport zu treiben.

In der PACT-Gruppe wurde dieses Ziel erheblich häufiger erreicht als in der Kontrollgruppe ( $70,4 \%$ vs. $43,4 \%$; $\mathrm{p}=$ 0,001 ). Weitere Analysen weisen auf eine signifikante Verbesserung der kardiovaskulären Reservekapazität durch PACT hin: Der Quotient aus den $\mathrm{VO}_{2 \text { peak }}{ }^{-}$ Werten der beiden Gruppen lag bei 1,04 zugunsten der PACT-Gruppe $(\mathrm{p}=0,032)$.

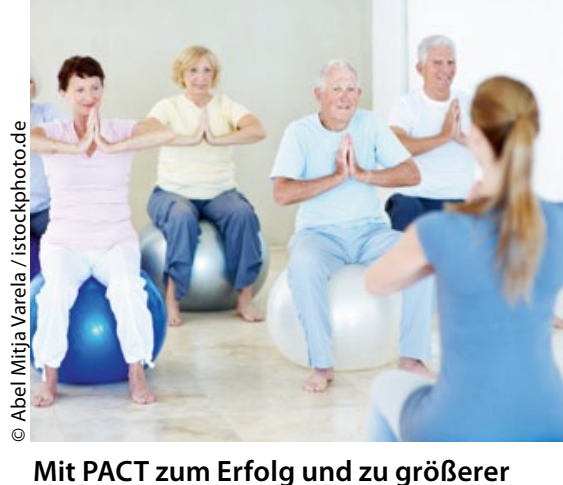

Mit PACT zum Erfolg und zu grö
kardiorespiratorischer Fitness.

Signifikante Unterschiede zwischen den Gruppen zeigten sich auch für Muskelstärke ( $p<0,001)$, Auftreten von Depressionen $(\mathrm{p}=0,020)$ und allgemeine psychische Gesundheit $(\mathrm{p}=0,040)$.

Fazit: Ein 12-monatiges Trainingsprogramm mit begleitender psychologischer Betreuung kann Patienten nach einer Krebsbehandlung zur körperlichen Aktivität anhalten und scheint sich auch messbar auf die kardiovaskuläre Reservekapazität auszuwirken. Christina Berndt

Midtgaard J et al. Efficacy of multimodal exercise-based rehabilitation on physical activity, cardiorespiratory fitness, and patient-reported outcomes in cancer survivors: A randomized, controlled trial. Ann Oncol. 2013 May 23. [Epub ahead of print].

\section{Krebsrisikos bei BRCA1- und BRCA2-Mutationen}

\section{Trägerinnen von Mutationen in den Tumorsuppressor-Genen BRCA1 und} BRCA2 entwickeln signifikant häufiger Mamma-, Ovarial- oder kontralaterale Mammakarzinome als Frauen ohne diese genetische Prädispositon. Bisher blieb die exakte Risikohöhe in Abhängigkeit von verschiedenen Variablen wie Alter, Familienanamnese oder Positivität für Suszeptibilitäts-Allele unklar.

\footnotetext{
$\mathrm{n}$ den meisten Penetranzstudien mit Trägerinnen von pathogenen BRCA1und/oder BRCA2-Mutationen wurde ein retrospektives Design benutzt und die Forscher kamen in ihrer Risikoabschätzung für das Mamma- oder Ovarialkarzinom zu höchst unterschiedlichen Ergebnissen mit einer großen Bandbreite. Das Krebsrisiko variiert in dieser Population in Abhängigkeit von Alter, familiärer Vorbelastung, Typ sowie Situs der Mutation und Lebensstilfaktoren. Auch das Vorhandensein verschiedener Suszeptibiltäts-Allele scheint das Erkrankungsrisiko von Frauen mit BRCAMutationen zu modifizieren.
}

Um das Risiko prospektiv abzuschätzen, griffen Nasim Mavaddat und Kollegen auf Daten der 1998 gestarteten und noch laufenden Studie EMBRACE (Epidemiological Study of BRCA1 and BRCA2 mutation carriers) zurück. Von 978 BRCA1- und 909 BRCA2-Mutationsträgerinnen aus Großbritannien waren zum Zeitpunkt des Studieneinschlusses 988 frei von Brustkrebs und 1.509 ohne Diagnose eines Ovarialkarzinoms. 651 Frauen litten bereits unter unilateralem Brustkrebs.

Das durchschnittliche kumulative Risiko im 70. Lebensjahr betrug bei BRCA1-Mutationen $60 \%$ für Brustkrebs,
$59 \%$ für das Ovarialkarzinom und $83 \%$ für das kontralaterale Mammakarzinom, bei BRCA2-Mutationen 55\%, 16,5\% und $62 \%$. BRCA2-Mutationen in der obersten Risikotertile plus positivem Nachweis von Mammakarzinom-Suszeptibilitäts-Allelen erhöhten signifikant das Brustkrebsrisiko.

Fazit: Prospektive Risikoanalysen bestätigen die positive Korrelation von BRCA1-, BRCA2-Mutationen und der Entwicklung von Brustkrebs, Ovarialkarzinomen oder einem kontralateren Mammakarzinom. Sie zeigt darüber hinaus aber auch, dass gerade die Kombination verschiedener Mammakarzinom-Suszeptibiltäts-Allele mit BRCA2Mutationen prädiktiv für das Brustkrebsrisiko sind.

Wolfgang Zimmermann

Mavaddat $\mathrm{N}$ et al. Cancer risks for BRCA1 and BRCA2 mutation carriers: results from prospective analysis of EMBRACE. J Natl Cancer Inst 2013;105(11):812-22. 